Musées, Patrimoine et Culture scientifiques et techniques

$137 \mid 2011$

septembre - octobre 2011

\title{
Homme-femme : un projet collaboratif
}

Man-Woman: a collaborative project

\section{Lucile Guittienne et Marlène Prost}

\section{OpenEdition \\ Journals}

Édition électronique

URL : http://journals.openedition.org/ocim/961

DOI : 10.4000/ocim.961

ISSN : 2108-646X

Éditeur

OCIM

Édition imprimée

Date de publication : 1 septembre 2011

Pagination : 19-25

ISSN : 0994-1908

Référence électronique

Lucile Guittienne et Marlène Prost, «Homme-femme : un projet collaboratif », La Lettre de I'OCIM [En

ligne], 137 | 2011, mis en ligne le 01 septembre 2013, consulté le 14 novembre 2019. URL : http://

journals.openedition.org/ocim/961 ; DOI : 10.4000/ocim.961 


\section{Homme-femme : \\ un projet collaboratif}

\section{Lucile Guittienne et Marlène Prost *}

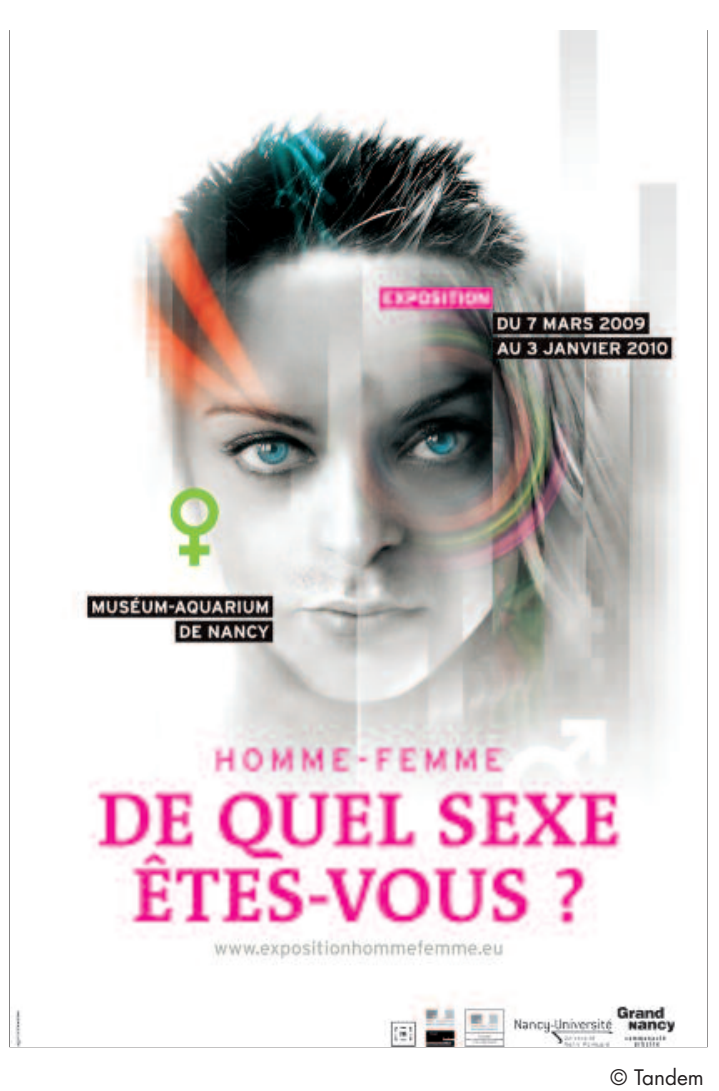

* Lucile Guittienne est directrice adjointe du Muséum-Aquarium de Nancy, attachée de conservation du patrimoine lucie.guittienne@grand-nancy.org Marlène Prost est responsable du département des Publics au Muséum-Aquarium de Nancy, attachée de conservation du patrimoine marlene.prost@grand-nancy.org
Proposée par le Muséum-Aquarium de Nancy en 2009, l'exposition « Homme-femme : de quel sexe êtes-vous? » a été conçue et animée grâce à une collaboration entre plusieurs structures issues notamment $d u$ milieu associatif, de l'Éducation nationale, de l'université de Nancy et de la communauté d'agglomération du Grand Nancy : les responsables du projet mettent ici en évidence les différents principes qui ont guidé cette démarche.

Le Muséum-Aquarium de Nancy est un muséum d'Histoire naturelle spécialisé en zoologie, il n'est ni un musée de société, ni un musée d'art ou un musée d'ethnographie. Pourtant, en 2009 il a réalisé une exposition sur le genre, non pas le genre taxonomique mais le genre sexuel : Homme-femme: de quel sexe êtes-vous?

Très rapidement, la complexité du sujet et ses implications sont apparues dans de nombreux domaines (art, religion, histoire, linguistique...). Ces thèmes délicats et complexes ne devaient pas être traités trop rapidement ni partiellement ; cependant, la taille de nos salles d'exposition $\left(350 \mathrm{~m}^{2}\right)$ ne le permettait pas. La solution est alors apparue évidente, il fallait se rapprocher d'autres structures plus spécialisées dans ces domaines pour, à la fois aborder les questions du genre dans leur intégralité, et offrir à nos visiteurs d'autres points de vue sur ce vaste sujet.

La démarche de travail en coopération qui en a découlé, marque la volonté d'inscrire ce projet sur un territoire, celui du Grand Nancy, l'une des deux tutelles du Muséum-Aquarium. Au-delà, il a aussi permis de fédérer plusieurs chercheurs locaux, mettant ainsi en valeur l'autre tutelle de l'établissement, l'université Henri Poincaré. 
Naître fille ou garçon. Être une femme ou un homme. L'appartenance à l'une de ces catégories est-elle suffisante pour caractériser un individu ? Cette question d'apparence simple, voire simpliste, a été au centre de l'exposition du Muséum-Aquarium de Nancy: Homme-femme : de quel sexe êtes-vous? présentée du 7 mars 2009 au 3 janvier 2010. Les questions autour du genre ont été abordées sans tabou et sans démagogie, tentant par là-même de dépasser le modèle homme versus femme.

Dans cette exposition, le visiteur était tout d'abord confronté à une observation des différences physiques externes et internes entre homme et femme puis subrepticement, le discours l'écartait de ses idées préconçues en abordant le sexe d'un point de vue sociologique, culturel ou encore linguistique. Conduit face à une multitude d'identités, le visiteur voyait s'éloigner le modèle dual classique. Disparu le moule préétabli en fonction de son sexe, chaque individu fait ses propres choix, s'invente son identité qui le distingue des autres. Il devient alors peu aisé de classer l'ensemble de l'humanité en deux catégories : homme ou femme.

Cette exposition centrée sur l'espèce humaine s'est construite comme un message de tolérance et d'ouverture vers le différent, le non soi. Dans ce cadre, des disciplines diverses furent

abordées : biologie au sens large (embryologie, biologie du développement, anatomie, génétique, écologie évolutive, sociologie, psychologie, philosophie, linguistique, droit...).

Face à une telle problématique, il n'était pas évident de choisir le bon titre, il devait interpeller le public visé et mettre en évidence toute la complexité du sujet. Le choix a été fait de mettre le visiteur en position de questionnement sur sa propre nature, lui montrant ainsi dès la lecture du titre, que rien n'est aussi simple que l'on pourrait le penser a priori

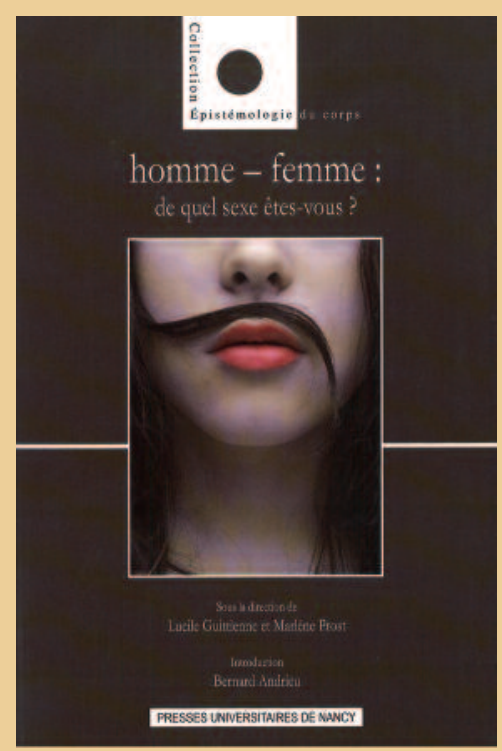

Le catalogue de l'exposition ○) Muséum-Aquarium de Nancy
Homme-femme : de quel sexe êtes-vous?, c'est donc l'histoire d'un projet touffu autour d'une exposition fédérant plusieurs partenaires qui ont développé une programmation culturelle collaborative.

\section{Le projet Homme-femme}

\section{Situer le projet dans son environnement}

Les " gender studies » apparaissent aux États-Unis au début des années 1970. Cette problématique de recherche est axée non pas sur le sexe mais sur le genre, terme difficilement traduisible en français : il se rapporte aux caractéristiques psychologiques et aux caractéristiques sociales créées par la culture humaine (M.-W. Matlin, 2007).

L'émergence de cette discipline en France a été beaucoup plus tardive et il a fallu attendre 2011 pour qu'un enseignement obligatoire lui soit consacré à Sciences Po Paris. Peu d'expositions portent clairement sur ce sujet (2 ou 3 choses que j'ignore d'elles, 2007, FRAC Lorraine) et les musées d'Histoire naturelle, lorsqu'ils traitent de la thématique, se cantonnent souvent à la différence des sexes chez les autres animaux (Parades ou la séduction dans le monde animal, 2005-2006, Muséum national d'Histoire naturelle). Ce sont bien souvent les artistes, telle Annette Messager, qui par le biais de différentes créations, ont révélé ces concepts au grand public.
Le Muséum-Aquarium choisit pourtant en 2009 de s'intéresser à cette problématique en centrant le propos sur l'espèce humaine. Aborder cette thématique, plutôt ancrée sciences humaines, dans un muséum d'Histoire naturelle peut dérouter le visiteur, plus habitué à découvrir dans ces lieux de culture scientifique les mœurs du gorille que ceux de son cousin l'humain. Une fois les réticences levées, celles du personnel et des élus, il convenait de cibler correctement ce sujet vaste, complexe et pluridisciplinaire et s'appuyer sur des formes d'expression variées pour compléter le propos : danse, chant, peinture, sculpture...

Dans ses expositions, le Muséum-Aquarium de Nancy cherche à privilégier l'objet patrimonial sur le discours que nous voulons plus discret. Dans ce cas précis, certaines sous thématiques comme « religion et genre » ne pouvaient pas être traitées trop succinctement sous peine de simplifier le propos, le rendant par là même non conforme à la réalité, voire sujet à polémique. D'autres médias, comme le livre ou le film, se prêtaient plus à l'argumentation et au développement de tels concepts.

Ainsi, Homme-femme : de quel sexe êtes-vous? propose une exposition, un catalogue, une programmation culturelle et un film : il réunit 40 partenaires différents, dont 15 seulement appartenaient déjà au réseau régulier du MuséumAquarium (1). 
importants étaient à l'initiative du service culturel de la Ville de Nancy, avec une équipe de travail et un budget dédiés. Homme-femme est le premier projet d'ampleur mené par le Muséum-Aquarium autour d'une de ses expositions.

Le principe de base du volet « activités culturelles » se fonde sur la coopération entre plusieurs institutions. Par coopérer, on entend agir et travailler conjointement avec autrui. On peut même parler de travail collaboratif, s'agissant d'un processus animé par des acteurs qui s'attaquent aux différents aspects d'un problème, exploitent leurs différences et cherchent à mettre en œuvre des solutions communes (M.-J. Couchaere, 2011).

\section{Les objectifs de cette programmation culturelle partenariale}

Dans l'esprit des organisateurs, mettre en place une programmation culturelle collaborative visait à offrir une vision la plus large possible sur la thématique du sexe et du genre chez l'Homme. Le partenaire devait se saisir du thème pour le faire sien avec ses propres outils (4). L'appropriation du sujet allait jusqu'à une intégration dans sa programmation de saison et jusqu'à une prise en compte complète de la part financière. La conception, la réalisation et le financement des supports de communication pour diffuser cette programmation étaient entièrement assumés par le Muséum-Aquarium. Il s'agissait d'un site Internet dédié au projet global, d'un agenda culturel semestriel regroupant l'ensemble des manifestations proposées et de totems de communication, estampillés Homme-femme et installés sur chacun des sites au moment de l'événement.

Aucune barrière n'a été posée lorsqu'il s'est agi de contacter les partenaires potentiels. L'objectif était d'élargir au maximum la vision sur cette thématique et d'inciter le public fidélisé de ces établissements à en découvrir d'autres. Tous les musées, médiathèques, galeries d'art, centres d'art, DRAC, FRAC, théâtres, opéra, ballet, cinémas, écoles supérieures, librairies, conservatoire de musique, associations, festivals locaux, service de culture scientifique et technique, de Nancy et alentours, ont été contactés pour solliciter leur participation. Ceux qui ont manifesté leur intérêt, ont ensuite été rencontrés individuellement puis collectivement.

Ces échanges menés à différentes échelles (en tête à tête et en groupe) étaient indispensables pour favoriser le dialogue et éviter toute incompréhension. Dans notre cas précis, le rendez-vous individuel a manqué pour l'une des collaborations et c'est justement sur celle-ci que nous avons rencontré quelques difficultés dues à un déficit de communication. Ce projet imparfait a permis de clarifier les échanges suivants, les coordinatrices ayant pris la mesure de l'importance de ces rencontres individualisées. Une première critique peut être apportée à ce stade. En cherchant un appui politique auprès d'une des tutelles du
Muséum-Aquarium, cette démarche de collaboration aurait pu être présentée comme un événement culturel fort sur le territoire de Nancy. Porté par un élu, il aurait gagné en reconnaissance (à l'égard des autres collectivités et des structures sollicitées) et aurait peut-être permis de faciliter l'obtention de financements supplémentaires.

\section{L'organisation du travail}

La réunion générale réunissant une partie des partenaires a constitué un moment fort de cette collaboration : vingt établissements de l'agglomération marquaient leur intérêt à travailler ensemble autour d'un projet commun. Il s'est agi de présenter les coordinatrices/interlocutrices, l'ensemble du projet (exposition, film, activités culturelles) et les « règles du jeu », autrement dit, ce qui était attendu des personnes présentes. Le tour de table, qui a suivi, a conduit chacun à préciser sa contribution : le musée des Beaux-Arts a proposé un cycle de visites commentées sur le thème, le ballet de Lorraine un spectacle dans l'un des jardins de la ville, l'artiste Mu a présenté ses œuvres dans une galerie d'art... En quittant la réunion, chaque contributeur savait qu'il aurait à fournir pour une date donnée, des éléments de présentation précis destinés à alimenter l'agenda culturel.

Cette réunion a permis l'organisation de projets croisés, favorisés par la rencontre de partenaires qui ne se connaissaient pas jusqu'alors. C'est justement ce champ d'autonomie que les coordinatrices souhaitaient favoriser : que les partenaires se saisissent du projet, se l'approprient et l'assimilent selon leurs spécificités.

Des travaux d'étudiants en design ont été présentés sous la forme d'une exposition dans une médiathèque et un DVD de portraits filmés réalisés par des étudiants en art a été diffusé dans tous les musées de la ville à l'occasion

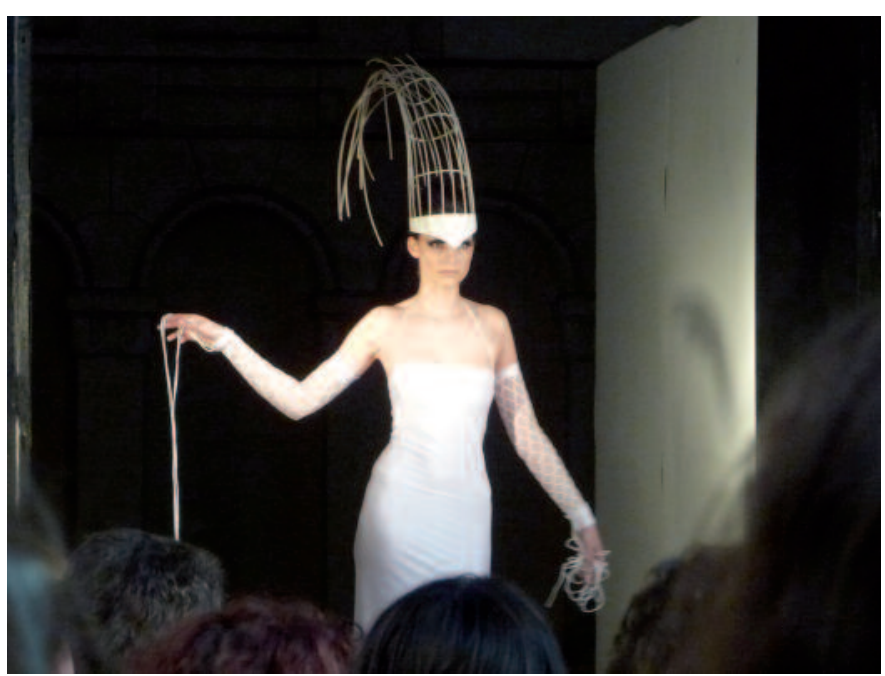

"Dialogue », défilé de mode, créations vestimentaires sur le thème homme-femme par les élèves de la section design de mode à l'école de Condé de Nancy (c) Muséum-Aquarium de Nancy 


\section{La politique d'exposition du Muséum-Aquarium de Nancy}

Comme c'est le cas pour bon nombre d'autres muséums, le rassemblement de collections de cabinets de curiosités au XVIIIe siècle a abouti à la création d'un cabinet d'Histoire naturelle de Nancy. C'est sous l'impulsion de Lucien Cuénot, naturaliste et biologiste nancéien, nommé directeur de ce cabinet en 1898, que le muséum va prendre sa forme actuelle. L'enrichissement des collections va provoquer leur éclatement par discipline et seule la zoologie et une partie de la paléontologie trouveront leur place dans le musée de Zoologie créé en 1933.

Le Muséum-Aquarium de Nancy est un être étrange et bicéphale, ce qui n'est pas sans complication. Dans les années 1970, des bacs d'aquariums tropicaux sont peu à peu construits sur l'ensemble du rez-de-chaussée. L'établissement comprend actuellement 57 aquariums de présentation au public. Mêlant le vivant et des collections patrimoniales, l'aspect muséal classique et le parc zoologique, cet établissement se voit doté en 1998 d'un nom biculturel : Muséum-Aquarium. Mais, la dualité de cet établissement ne réside pas seulement dans son nom ou dans ses présentations, elle est dans sa nature même. Il est l'un des rares sur le territoire national à posséder une double tutelle unissant une collectivité, la Communauté urbaine du Grand Nancy, et une université, l'université Henri Poincaré.

Après de premiers aménagements dans les galeries d'aquariums dans les années 1970, c'est soixante-dix ans après sa création que l'établissement vit sa seconde jeunesse. Des réserves sont créées, une nouvelle muséographie de l'exposition permanente est présentée en 2005, libérant ainsi deux espaces d'exposition dédiés aux événements temporaires.
Depuis cette rénovation, le Muséum-Aquarium de Nancy a développé une véritable politique d'expositions temporaires. Ainsi, une exposition majeure entièrement conçue en interne est présentée chaque année. Les thèmes se veulent volontairement interpellants, voire impertinents : les déjections Crotte alors! en 2006, le sexe Homme-femme : de quel sexe êtes-vous? en 2009. La démarche reste toujours pluridisciplinaire, n’hésitant pas à mêler l'art aux sciences : Salon des artistes animaliers en 2007, 2 moi -2 artistes en 2008, De crépuscule en crépuscule en 2011. Quelquefois, les thématiques suivent des programmes de plus grande ampleur, Luxembourg et Grande Région capitale européenne de la culture, Les envahisseurs en 2007, l'année internationale de la biodiversité, Biodiversité : hommage à une limace disparue en 2010.

Depuis 2010, le Muséum-Aquarium travaille sur son projet scientifique et culturel. Cela a permis de dégager clairement la ligne directrice de la programmation culturelle. L'approche paléontologique et zoologique, en considérant l'Homme comme un animal à part entière, est favorisée tout en conservant une ouverture vers les sciences humaines et l'art. Chaque année, une exposition temporaire sera donc programmée, elle durera environ 9 mois et son pu blic cible sera clairement défini (très jeune public, jeune public, adolescent, adulte, senior...) et variera selon les années. Cela permettra d'éviter de tenter, en vain, de programmer des expositions dites tout public. Ces expositions devront être dans la lignée des précédentes, originales et un peu décalées. De plus petites expositions (1 ou 2 par an) à destination d'un public plus spécialisé compléteront la programmation en mettant en valeur, à travers une scénographie simple, des pièces de nos collections. de la Nuit européenne des musées. Le Muséum-Aquarium a aussi pu à cette occasion développer un projet avec un nouveau partenaire européen, rencontré quelques mois auparavant lors d'une réunion d'échange avec les musées de Karlsruhe, ville jumelée avec Nancy : dans un premier temps, l'exposition a été présentée sous forme de conférence à l'institut français de Karlsruhe dans le cadre du festival Perspectives de femmes 2009 et dans un second temps, l'organisatrice allemande est venue présenter une conférence sur les femmes et l'Iran, pays à l'honneur de cette édition.

Dans cette réunion générale se cristallisaient donc plusieurs éléments qui ont conditionné la bonne poursuite du projet. Un premier tour de table a permis de situer chacun des contributeurs et sa structure. La présentation précise du projet a posé les bases de la coopération, évoqué les résultats attendus et les échéances à respecter. Ces échanges ont aussi été l'occasion de préciser la place de chacun dans le projet afin que tous - coordinateur et contributeurs puissent mesurer leur marge de manœuvre. Le projet était coordonné par deux personnes du Muséum-Aquarium. Cette organisation a permis de pallier les problèmes d'absence ou d'indisponibilité : les contributeurs trouvaient ainsi toujours une interlocutrice en mesure de leur répondre.

Une autre critique peut être présentée à ce niveau. Une seconde réunion générale avant le lancement du travail sur l'agenda culturel aurait permis de consolider les liens entre tous les contributeurs. Cela aurait pu se traduire, par exemple, par un bilan à mi-parcours de la fréquentation des activités, un relevé des dysfonctionnements... C'est seulement dans le dernier mois de l'exposition qu'une soirée 
bilan conviviale a été proposée, mêlant visite de l'exposition, projection du film et retour sur la programmation culturelle. De façon plus formelle enfin, les contributeurs ont tous reçu le bilan complet du projet Homme-femme. Ce document détaillait entre autres, la fréquentation de l'exposition, de la médiation culturelle organisée autour et de la programmation culturelle (dans et hors les murs), les retombées dans la presse, les autres supports de communication et les subventions obtenues.

\section{D'autres projets collaboratifs}

Le projet Homme-femme n'est pas un cas isolé, le Muséum-Aquarium prend part à d'autres projets collaboratifs qui perdurent parfois depuis plusieurs années.

Depuis dix ans maintenant, les six musées de l'agglomération nancéienne organisent conjointement une nocturne étudiante au printemps : 1 muit-6 musées-47 000 étudiants. Le pilotage de la manifestation est tournant. Les rôles sont ici différents du projet Homme-femme dans le sens où d'une année à l'autre, chaque musée est plus ou moins pris par l'organisation mais néanmoins partie prenante dans toutes les décisions. Il est intéressant de noter que plusieurs groupes d'étudiants, dans le cadre de leur cursus, interviennent désormais dans l'organisation. À chaque fois, le musée-pilote

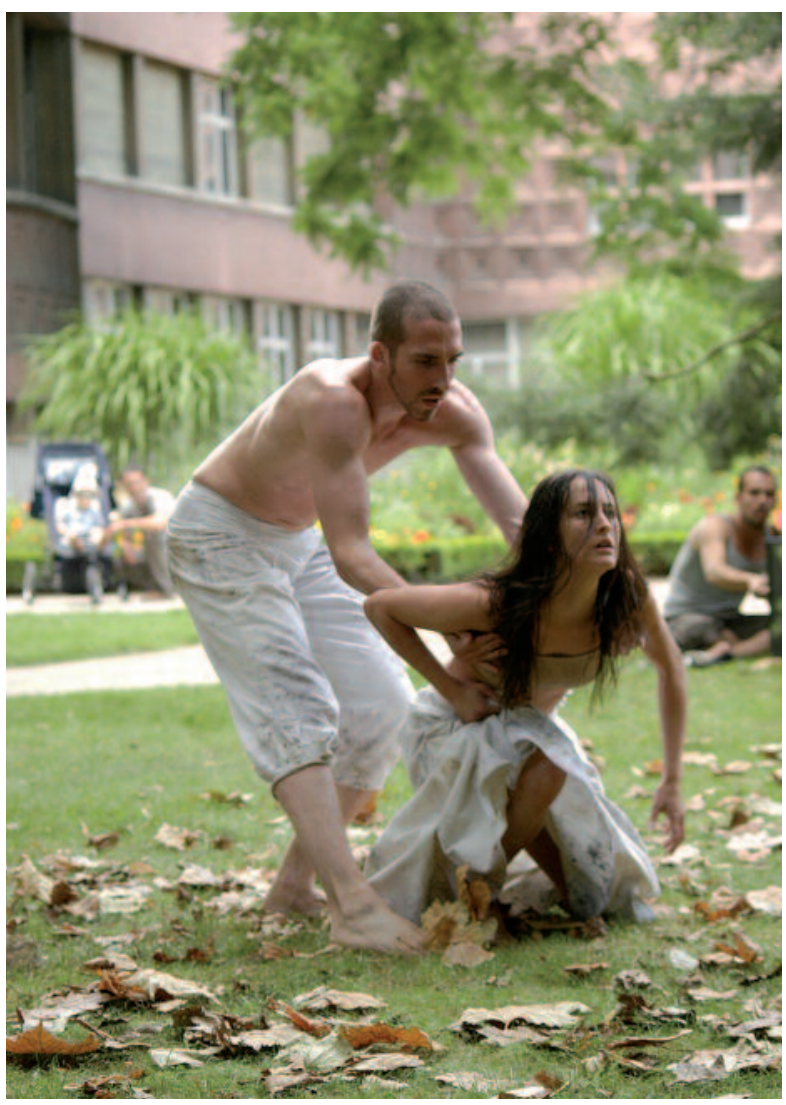

"Le Jardin », pièce dansée présentée par le CCN-Ballet de Lorraine. (๑) Muséum-Aquarium de Nancy accompagne le travail des étudiants tout au long de l'année, de sorte qu'il reste en cohérence avec l'esprit voulu par les membres. À des moments précis, ce sont les six structures qui viennent à la rencontre des étudiants pour échanger sur leur travail.

Dans ce cas, l'ancienneté du projet rend les échanges plus aisés dans le sens où tous les participants ont, au fil des années, appris à se connaître et ont l'habitude de travailler ensemble.

Plus récemment, d'autres projets dans le domaine de la culture scientifique et technique ont vu le jour. Citons deux exemples : Les Conf'curieuses, un cycle de conférences scientifiques mensuelles organisé par le MuséumAquarium, les Conservatoire et Jardins Botaniques de Nancy et le service de culture scientifique et technique du PRES de l'université de Lorraine et La science au coin $d u$ bois, une manifestation en forêt proposée le dimanche 26 juin par le Muséum-Aquarium, les Conservatoire et Jardins Botaniques de Nancy, le service de culture scientifique et technique du PRES de l'université de Lorraine, l'INRA, l'ONF et AgroParisTech.

Unir plusieurs structures pour concevoir un projet est certes séduisant. La mise en commun de compétences et de moyens, humains et financiers, permet l'élaboration d'une manifestation de plus grande ampleur. Toutefois, selon le nombre d'intervenants et surtout selon la qualité du pilotage et la répartition des tâches à accomplir, la collaboration est plus ou moins fructueuse et chronophage.

\section{Conclusion}

Aucune véritable étude de public n’a pu être menée autour de ces activités culturelles proposées autour du projet Homme-femme : de quel sexe êtes-vous? Toutefois, les données chiffrées permettent de juger de leur importance : 6800 personnes ont suivi un des 82 rendez-vous proposés dans les locaux du Muséum-Aquarium ou dans une des structures partenaires.

Organiser un évènement de cette ampleur au niveau local, régional et européen a permis au Muséum-Aquarium de se positionner en tant qu'établissement culturel de référence. Les muséums sont souvent considérés avant tout comme des lieux de science et non comme des lieux de culture. À cet égard, le rapprochement avec des structures culturelles plus classiques (musée des Beaux-Arts, opéra, ballet, médiathèque) a donc été bénéfique en terme d'image.

Nous avons qualifié ce projet de collaboratif. Cependant, un terme nouveau semble tout à fait correspondre : la proopération (J. Barrand). Il désigne une coopération cherchant à satisfaire tous les acteurs qui coopèrent en ayant le souci de produire des résultats durables pour toutes les parties, la question du durable dans la coopération entre institutions étant cruciale aujourd'hui. 


\section{Notes}

(1) Association Club Soroptimist, Association femmes et sciences, Association femmes et mathématiques, Association Lesbienne, Gay, Bisexuelle et Transexuelle, Ballet national de Lorraine, Bibliothèque des Sciences et Techniques, Bibliothèque municipale de Saint Max, Carrefour Santé, Ville de Nancy, Centre d'art contemporain la synagogue de Delme, Centre départemental de Documentation pédagogique, Centre d'Information du Droit des Femmes et des Familles, Conservatoire régional du Grand Nancy, Collège Jean Lurçat, Conservatoire régional de l'Image Nancy Lorraine, Conservatoire et Jardins Botaniques de Nancy, CNRS, École de Condé de Nancy, École nationale supérieure des BeauxArts de Nancy, Équinoxe Nancy Lorraine, Festival du film de chercheur, Forum-IRTS de Lorraine, Galerie Ovadia, Groupe Sciences Innovation et Société (Nancy), Librairie La Parenthèse, Librairie L’Autre Rive, Lycée technique Henri Loritz, Materia Prima / le TOTEM, MSH Lorraine, Médiathèque de Nancy, Médiathèque Haut du Lièvre, Médiathèque Jules Verne de Vandœuvre-lès-Nancy, Mission départementale aux droits des femmes et à l'égalité, Musée de l'Image à Épinal, Musée des BeauxArts de Nancy, Musée lorrain, Rectorat Nancy-Metz, Service de Culture scientifique et technique de l'université Henri Poincaré, Théâtre Gérard Philipe de Frouard, Ville de Karlsruhe, Vidéoscop.

(2) Bernard Andrieu, professeur en épistémologie du corps et des pratiques corporelles, UHP, ACCORPS/Archives Poincaré, CNRS ; Christian Dournon, professeur de biologie du développement et de la reproduction, Nancy Université.

(3) Canal U : www.canal-u.tv/producteurs/les_amphis_de_france_5/dossier_ programmes/sociologie/hommes_femmes_sortir_des_stereotypes.

Le coin des vidéos du site psychologie sociale : www.psychologie-sociale. com/index.php?option=com_content\&task=view\&id=420\&Itemid=124

(4) Le théâtre Gérard Philippe de Frouard, par exemple, avait déjà en projet de programmer une pièce évoquant les questions de la famille et du genre. Le directeur en a alors profité pour proposer en parallèle, une rencontre autour de la question du genre dans les représentations théâtrales jeune public.

\section{Bibliographie}

Barrand, J. Le Manager agile : vers un nouveau management pour affronter la turbulence. Dunod, Paris, 2009.

Couchaere, M.-J. Favoriser le travail en équipe par la coopération. Esf, 2011

Dhume-Sonzogni, F. La coopération dans l'action publique. L'Harmattan, 2006.

Boutin, G. et Le Cren, F. Le partenariat : entre utopie et réalité. Nouvelles éditions, 2004.

Guittienne, L. et Prost, M. (dir.). Homme-femme : de quel sexe êtes-vous? PUN, 2009

Matlin, M. W. Psychologie des femmes. De Boeck, 2007.

Homme-femme : sortir des stéréotypes, DVD. Vidéoscop, 2009

Canal U : www.canal-u.tv/producteurs/les_amphis_de_france_5/dossier_ programmes/sociologie/hommes_femmes_sortir_des_stereotypes 